\title{
Diseño y validación de la Escala de Actitudes hacia los Cuidados Paliativos de un Familiar
}

\author{
Design and validation of the Scale of Attitudes \\ towards Palliative Care of a Relative
}

\author{
Tatiana Pedroza Varela, Nicolás Garzón Rodríguez, Paola Ortíz Bello, \\ Daniel Murcia Leal y Fernando Riveros Munévar
}

\author{
Universidad de La Sabana $^{1}$
}

Autor para correspondencia: Fernando Riveros Munévar, edgar.riveros@unisabana.edu.co.

\section{RESUMEN}

\begin{abstract}
El presente estudio tuvo como objetivo diseñar y validar un instrumento que midiera las actitudes hacia los cuidados paliativos de un familiar. Inicialmente, se realizó una revisión teórica y se construyó una tabla de especificaciones según los componentes de las actitudes, cruzados con los elementos de los cuidados paliativos. Después, tras un proceso de validación por jueces expertos y una prueba piloto, se aplicó el instrumento a 140 ciudadanos colombianos con una edad promedio de 29.05 años, tras de lo cual se efectuó un análisis factorial exploratorio de los datos obtenidos. Hecha la validación, se obtuvo un coeficiente general de consistencia interna de .851, y se concluyó con la elaboración de un instrumento conformado por 16 reactivos que se califican en una escala Likert de cuatro puntos distribuidos en tres factores (valoración general positiva del cuidado, compromiso con el cuidado y valoración general negativa del cuidado), modelo que fue sometido a un análisis factorial confirmatorio que mostró una adecuada armonía. El instrumento así elaborado posee validez y confiabilidad considerables, y es útil para medir las actitudes hacia los cuidados paliativos de un familiar.
\end{abstract}

Palabras clave: Actitudes; Cuidados paliativos; Psicometría.

\begin{abstract}
This study aimed to design and validate a scale to measure attitudes towards the palliative care of relatives. After reviewing the research literature, a table with the corresponding specifications included how the attitudes crossed with the components of palliative care. After a process of validation by expert judges and a pilot test, a total of 140 Colombian citizens (Age $M \pm S D=29.05 \pm 12.180)$ responded to the instrument. After an exploratory factorial analysis of the data, the evaluation process yielded a general internal consistency value of .851. The final scale consisted of 16 items with four Likert options each, composing three factors: a) overall positive assessment of care, $b$ ) commitment to care, and c) general negative assessment of care. A confirmatory factor analysis revealed adequate consistency of the model. The instrument has high validity and reliability to measure attitudes toward palliative care of a family member.
\end{abstract}

Key words: Attitudes; Palliative care; Psychometrics.

\footnotetext{
${ }^{1}$ Campus del Puente del Común, Km. 7, Autopista Norte de Bogotá, Chía, Cundinamarca, Colombia, tel: 573175122983 , correos electrónicos: tatianapeva@unisabana.edu.co,nicolasgaro@unisabana.edu.co, leidyorbe@unisabana.edu.co,danielmule@unisabana.edu.co y edgar.riveros@ unisabana.edu.co.
} 
Recibido: $14 / 04 / 2020$

Aceptado: 04/08/2020

$\checkmark$ $\mathrm{n}$ las ciencias de la salud existe una amplia variedad de enfermedades crónico degenerativas que desembocan generalmente en un diagnóstico terminal (Grau et al., 2008); el cual ha adquirido importancia en el último siglo debido a que se hace cada vez más frecuente en los centros asistenciales (Ascencio, Allende y Verástegui, 2014). Por consiguiente, las implicaciones para los profesionales de la salud y los familiares del paciente respecto al cuidado y atención del enfermo han despertado el interés de varios investigadores (Ascencio et al., 2014; Bermejo, Villacieros, Carabias, Pichardo y Diner, 2013; Marchán, 2016; Sánchez y Díaz, 2013; Sánchez et al., 2017; Sarmiento, 2015, entre muchos otros). Según el Observatorio Colombiano de Cuidados Paliativos (2016), en el año 2016 murieron 136,846 personas por condiciones crónicas que eran susceptibles de cuidados paliativos, y únicamente $0.3 \%$ de esta población (346 personas) recibió atención en dichos cuidados; además, solo 3 de 57 facultades de medicina incluyen los cuidados paliativos en sus programas (Pastrana, de Lima y Wenk, 2015), lo que denota la considerable necesidad de profesionales especializados en esa área (Grau et al., 2008).

Los cuidados paliativos son las actividades destinadas a brindar recursos físicos y emocionales a los acompañantes y al paciente para que este curse de la mejor manera posible su enfermedad (Grau et al., 2008). Así pues, los cuidados paliativos se definen como el apoyo y acompañamiento activo a pacientes próximos a la muerte con alguna enfermedad en fase terminal, es decir, que ya no responde a los tratamientos curativos, siendo entonces primordiales el control y alivio del dolor y de otros síntomas de origen psicológico, social y espiritual (Gómez, Clemente, Bugallo y Gandoy, 2016; López, 2015; Tejeda, 2011). Asimismo, también es importante el manejo paliativo guiado por una primera evaluación completa del estado y de las necesidades biopsicosociales del paciente a fin de determinar su manejo más apropiado (Ferrer, Padgett y Ellis, 2016; López, 2015).
Cabe mencionar que los cuidados paliativos se enfocan en apoyar la calidad de vida del paciente y mejorar los síntomas y el estrés inherentes a una enfermedad terminal; para ello, se trabaja sobre unos pilares fundamentales: la toma de decisiones, la experiencia emocional compartida, la vinculación social, el tiempo de cuidado y la logística, toda vez que el cuidado paliativo "implica ver la vida de una manera diferente, modificar las funciones a las que se está acostumbrado, tomar decisiones en medio de alternativas complicadas, asumir responsabilidades o realizar tareas y acciones de cuidado físico, social, psicológico y religioso para atender las necesidades cambiantes de la persona cuidada" (Flores, Rivas y Seguel, 2012; cf. también Tripodoro, Veloso y Llanos, 2015).

La toma de decisiones involucra aquellas determinaciones relacionadas con el proceso asistencial en curso que toman los pacientes, sus familiares y el personal asistencial, lo que implica la evaluación de los riesgos posibles (Ferrer et al., 2016). La experiencia emocional compartida corresponde a la carga emocional del cuidador y del enfermo, sean sentimientos positivos o negativos (Rodrigues, 2010), lo cual será de vital importancia en virtud de que la evolución psicológica del paciente depende de los posibles sentimientos de culpa, ansiedad o decepción de sus seres queridos respecto a "renunciar" a la búsqueda del tratamiento curativo (Ferrer et al., 2016). La vinculación social, a su vez, se concibe como las relaciones que componen el entorno social del paciente y que están presentes en cualquier proceso en el que este se involucre, por lo que repercuten en su vida personal y afectiva (Butow et al., 2007; Ferrer et al., 2016; Padgett y Ferrer, 2015). El tiempo de cuidado se refiere al tiempo que el cuidador comparte con el paciente y que puede ser completo o parcial (Rodrigues, 2010). Por último, la logística es la coordinación de servicios sociales o clínicos, los tratamientos y las propias necesidades del cuidador, además de las tareas físicas que involucran el cuidado (Rodrigues, 2010).

Dadas las condiciones anteriores, se puede afirmar que los cuidados paliativos ocurren en un contexto social, por lo que su valoración se vuelve natural (Padgett y Ferrer, 2015); no obstante, tal 
apreciación puede variar según el tiempo que el cuidador deba emplear para acompañar al paciente, teniendo en cuenta la enfermedad del mismo (Tejeda, 2011). Es importante señalar que las valoraciones se denominan actitudes y se definen como las evaluaciones globales y relativamente estables que los sujetos hacen sobre otras personas, ideas o cosas que técnicamente reciben la designación de objetos de actitud (Briñol, Falces y Becerra, 2007).

Las actitudes constan de tres componentes: el primero de ellos es el cognitivo, que incluye tanto los pensamientos como las creencias que la persona tiene sobre el objeto de actitud a partir de una relación directa o indirecta, o bien de los conocimientos previos que tiene sobre aquel. En segundo lugar se encuentra el elemento afectivo, el cual permite a la persona asociar sentimientos y emociones al objeto de actitud, lo que puede ocurrir a partir de la mera exposición; así, una persona que haya estado en contacto frecuente con los cuidados paliativos puede desarrollar diferentes actitudes dependiendo de la calidad de su experiencia. Por último, se halla el componente conductual, en el que se incluyen las intenciones, disposiciones a la acción y comportamientos dirigidos hacia el objeto de actitud; en otras palabras, la disposición e intención que se tiene en función de los cuidados paliativos aportan también a la construcción de la actitud frente a dicho proceso (Briñol et al., 2007). De este modo, las actitudes hacia los cuidados paliativos serán entendidas en el presente estudio como predisposiciones aprendidas y evaluaciones globales, positivas o negativas, del apoyo o acompañamiento activo a una persona que padece una enfermedad crónica, degenerativa e irreversible y que se encuentra próxima a la muerte (Briñol et al., 2007; Gómez et al., 2016; López, 2015; Tejeda, 2011).

Es importante resaltar que estudiantes de ciencias de la salud han evaluado el cuidado paliativo como un ejercicio de riesgo o como un oficio incómodo al verse indispuestos para la "transmisión de malas noticias, [el] manejo del duelo y [la] comunicación de una limitación o adecuación terapéutica" (Pastrana et al., 2015, p. 136). Con ello, se facilita la aparición del burnout, o síndrome del estrés laboral asistencial, definido por Maslach y Jackson (1981) como el "agotamiento emocional, despersonalización y realización personal reducida" (cf. también Gómez et al., 2016) que surgen como consecuencia de este acompañamiento y que conllevan una actitud por parte del cuidador. Cabe señalar que principalmente se han estudiado las actitudes de enfermeras y médicos ante el cuidado del enfermo (Espinoza, Luengo y Sanhueza, 2016), pero no se ha indagado sobre la influencia de las actitudes del núcleo familiar en dicho cuidado a partir de su nivel experiencial y del vínculo compartido con el enfermo (Arriaza, Cancino y Sanhueza, 2009).

La literatura especializada sigue el mismo curso que los instrumentos que evalúen este constructo. Hay instrumentos validados en poblaciones extranjeras que miden las actitudes de los profesionales, tales como la escala FATCOD, diseñada para medir las actitudes del personal de enfermería y adaptado en una población chilena por Espinoza et al. (2016), y la escala EACP, creada para medir las actitudes hacia la práctica de cuidados paliativos también en el personal de enfermería, validada en México por Ascencio (2015). No obstante, en la mencionada revisión no se encuentran instrumentos validados en muestras latinoamericanas que evalúen las actitudes de los familiares como cuidadores o potenciales cuidadores de dichos pacientes, aun cuando la familia es una parte esencial de la medicina paliativa debido a que constituye un foco principal de atención al lograr armonía y equilibrio en la fase terminal de la enfermedad (Tejeda, 2011). Dado que la mayoría de los cuidadores de pacientes con enfermedades crónicas son los mismos familiares, y que se desconoce un instrumento que pueda medir sus actitudes hacia el cuidado paliativo, se llevó a cabo el presente estudio, que tuvo como objetivo plantear y validar una herramienta que permitiese determinar las actitudes hacia los cuidados paliativos de un familiar en una muestra colombiana. En consecuencia, la presente investigación fue de tipo psicométrico instrumental en virtud de que está encaminada al desarrollo, diseño o adaptación de un instrumento, así como al estudio de sus propiedades psicométricas (Montero y León, 2005). De hecho, el ejer- 
cicio investigativo consistió en desarrollar una prueba para evaluar diversos componentes de las actitudes hacia los cuidados paliativos de un familiar.

\section{MÉTODO}

\section{Participantes}

Se contó con la participación de siete jueces expertos, tres de ellos especialistas en medición y evaluación psicométrica, y cuatro teóricos en el área de los cuidados paliativos; para el desarrollo de la prueba piloto se emplearon diez cuidadores potenciales (seis hombres y cuatro mujeres), con edades de entre 20 y 40 años, seleccionados por conveniencia, y para el análisis que permitió la estandarización de la prueba se dispuso de 140 participantes de una muestra colombiana, con una edad media de 29.05 (desviación típica \pm 12.18 ), de los cuales 59.3\% fueron mujeres. Se excluyó a aquellos participantes cuya respuesta de la primera pregunta, relativa al consentimiento informado, fue no estar de acuerdo con que sus resultados se utilizaran con fines académicos e investigativos de manera anónima, y también a los menores de 18 años a fin de resguardar los principios éticos de la investigación. Se accedió a todos los participantes para la prueba piloto, y para la aplicación por conveniencia el formulario se envió a través de las redes sociales.

Cabe señalar que ninguno de los participantes eran cuidadores activos al momento de la aplicación, criterio que se utilizó para evitar los sesgos acerca de la interpretación de sus actitudes hacia los cuidados paliativos.

\section{Procedimiento}

En primera instancia, se efectuó una revisión teórica sobre la actitud hacia los referidos cuidados, en la que se indagaron los componentes del constructo para el proceso de elaboración y distribución de los reactivos; para ello, se creó una tabla de especificaciones (Apéndice A) que incluía y cruzaba los componentes cognitivo, afectivo y conductual de las actitudes con las cinco áreas de los cuidados paliativos: toma de decisiones, experiencia emocional compartida, vinculación social, tiempo de cuidado y logística. Adicionalmente, se plantearon algunos ítems redundantes, es decir, aquellos que expresan la misma idea con una redacción mínimamente distinta, los que se emplean para evaluar la consistencia de las personas y aumentar la consistencia interna del instrumento (Ferrando y Anguiano, 2010; Lloret, Ferreres, Hernández y Tomás, 2014).

Una vez elaborado el instrumento, se construyeron 62 ítems que se sometieron al proceso de validación por los siete jueces expertos, a quienes se les pedía puntuar su pertinencia, coherencia, redacción y objetividad. Con base en lo anterior, se realizó la depuración de los ítems por medio del índice de validez de contenido de Lawshe (Apéndice $\mathrm{B}$ ) y el índice $\mathrm{W}$ de Kendall (Apéndice C) de concordancia entre jueces. Después, se efectuó la prueba piloto con el propósito de identificar problemas en la interpretación de los ítems; de este modo, se tomaron en cuenta las retroalimentaciones cualitativas para el refinamiento de los reactivos a fin de que fuesen comprendidos cabalmente.

La escala por validar contenía aspectos sociodemográficos, como edad, sexo y ocupación, además de los 37 reactivos resultantes de la depuración inicial. Este instrumento fue digitalizado $y$, una vez recogida la información, se procedió a invertir las puntuaciones de los ítems dependiendo de si medían una actitud positiva o negativa, y posteriormente se realizaron los análisis estadísticos por medio del programa SPSS ${ }^{\circledR}$, versión 23.

El análisis factorial exploratorio se refinó tras varios ensayos de reducción de factores mediante los cuales se buscaba que la carga de los ítems fuera clara respecto al factor (eliminando los ítems inapropiados para la prueba), y se desarrolló el análisis de consistencia interna a través del coeficiente alfa de Cronbach. Después, se efectuó el modelado por ecuaciones estructurales para confirmar el modelo obtenido. Por último, se elaboraron las normas de calificación de la escala, generando baremos mediante el método de rango (Apéndice E). 
Todo lo anterior se llevó a cabo respetando los principios éticos y el código de conducta de la American Psychological Association (1992), las leyes del país en que se realizó y los estándares éticos de la investigación, teniendo en cuenta que antes de participar se garantizó a cada persona el respeto a su dignidad, la protección de su bienestar y la privacidad de sus datos y respuestas.

\section{RESULTADOS}

En primer lugar, se realizó la tabla de especificaciones (Apéndice A), teniendo en cuenta los componentes cognitivo, afectivo y conductual de la actitud, así como las cinco áreas de los cuidados paliativos (toma de decisiones, experiencia emocional compartida, vinculación social, tiempo de cuidado y logística) para guiar el desarrollo de los ítems, los cuales una vez construidos fueron validados por los jueces (Apéndice B), de modo que 6 de los 62 ítems iniciales que puntuaron $r<0.6$ en el índice de validez de contenido de Lawshe se descartaron. Los ítems restantes se modificaron por la sugerencia cualitativa de los expertos. Adicionalmente, la evaluación de los jueces se sometió al análisis de validez de contenido $\mathrm{W}$ de Kendall, sin obtener concordancias entre ellos en ninguna de las dimensiones $(p<0.05)$ (Apéndice $\mathrm{C}$ ).

Para saber si era apropiado realizar el análisis factorial exploratorio, se desarrolló la prueba de Kaiser-Meyer-Olkin (KMO) y un análisis de esfericidad de Barlett, por los que se encontró que la muestra era suficiente y apropiada para ejecutar el análisis (KMO > .8; Barlett, $p<0.05)$. Paulatinamente se hizo una depuración para eliminar los ítems cuya carga no era notablemente específica a un factor, restando así un total de veinte ítems para el análisis.

Respecto a la varianza total acumulada de los tres factores hallados, el primero de ellos explicó $24.01 \%$ de la varianza, el segundo $22.56 \%$ y el tercero $11.37 \%$, de manera que en conjunto explicaban $56.49 \%$ de la varianza total acumulada de la escala, denominándose Valoración general positiva del cuidado, Compromiso con el cuidado y Valoración general negativa del cuidado, respectivamente.

Se ejecutó después el análisis de consistencia interna por factor (cuatro en total) a través del coeficiente alfa de Cronbach, el cual indicó que cuatro de los ítems se debían descartar dado que la consistencia interna de la prueba mejoraba al eliminarlos.

En la Tabla 1 se presenta el análisis factorial final (tres componentes) tras eliminar los ítems, para obtener un total de 16 reactivos que conforman la denominada Escala de Actitudes hacia los Cuidados Paliativos de un Familiar (Apéndice D), teniendo en cuenta que estos fueron reordenados según su numeración inicial.

Tabla 1. Análisis factorial final. Matriz de componentes rotada.

\begin{tabular}{|c|c|c|c|}
\hline \multirow{2}{*}{ Ítems } & \multicolumn{3}{|c|}{ Factores } \\
\cline { 2 - 4 } & FI & FII & FIII \\
\hline 10 & .820 & & \\
\hline 2 & .720 & & \\
\hline 8 & .704 & & \\
\hline 1 & .703 & & \\
\hline 14 & .687 & & \\
\hline 5 & .666 & & \\
\hline 9 & .560 & & \\
\hline 12 & & .756 & \\
\hline 16 & & .755 & \\
\hline 11 & & .702 & \\
\hline 13 & & .666 & \\
\hline 4 & & .617 & \\
\hline 7 & & .524 & \\
\hline 6 & & & .797 \\
\hline 15 & & & .708 \\
\hline 3 & & & .676 \\
\hline
\end{tabular}

La Tabla 2 muestra el análisis de fiabilidad por factor una vez finalizado el refinamiento de la escala.

Con el fin de verificar la idoneidad del modelo, se efectuó el análisis factorial confirmatorio por medio de modelos de ecuaciones estructurales. Dicho modelo se observa en la Figura 1, y los estadísticos correspondientes en la Tabla 3. 
Tabla 2. Análisis de fiabilidad de la escala.

\begin{tabular}{|c|c|c|c|c|}
\hline Factor & Ítems & $\begin{array}{l}\text { Correlación } \\
\text { ítem-factor }\end{array}$ & $\begin{array}{c}\text { Alfa } \\
\text { si se elimina }\end{array}$ & $\begin{array}{c}\text { Coeficiente } \\
\text { alfa }\end{array}$ \\
\hline \multirow{7}{*}{$\begin{array}{l}\text { I. Valoración } \\
\text { general positiva } \\
\text { del cuidado }\end{array}$} & 10 & 0.762 & 0.824 & \multirow[t]{7}{*}{0.863} \\
\hline & 2 & 0.595 & 0.849 & \\
\hline & 8 & 0.603 & 0.848 & \\
\hline & 1 & 0.552 & 0.855 & \\
\hline & 14 & 0.678 & 0.837 & \\
\hline & 5 & 0.635 & 0.843 & \\
\hline & 9 & 0.605 & 0.847 & \\
\hline \multirow{6}{*}{$\begin{array}{l}\text { II. Compromiso } \\
\text { con el cuidado }\end{array}$} & 12 & 0.660 & 0.747 & \multirow[t]{6}{*}{0.802} \\
\hline & 16 & 0.517 & 0.781 & \\
\hline & 11 & 0.558 & 0.772 & \\
\hline & 13 & 0.553 & 0.772 & \\
\hline & 4 & 0.558 & 0.771 & \\
\hline & 7 & 0.506 & 0.782 & \\
\hline \multirow{3}{*}{$\begin{array}{l}\text { III. Valoración } \\
\text { general negativa } \\
\text { del cuidado }\end{array}$} & $6^{*}$ & 0.529 & 0.328 & \multirow[t]{3}{*}{0.601} \\
\hline & $15^{*}$ & 0.348 & 0.592 & \\
\hline & $3 *$ & 0.365 & 0.568 & \\
\hline \multicolumn{4}{|l|}{ Alfa total } & 0.851 \\
\hline
\end{tabular}

Nota: * Ítems inversos.

Figura 1. Análisis factorial confirmatorio.

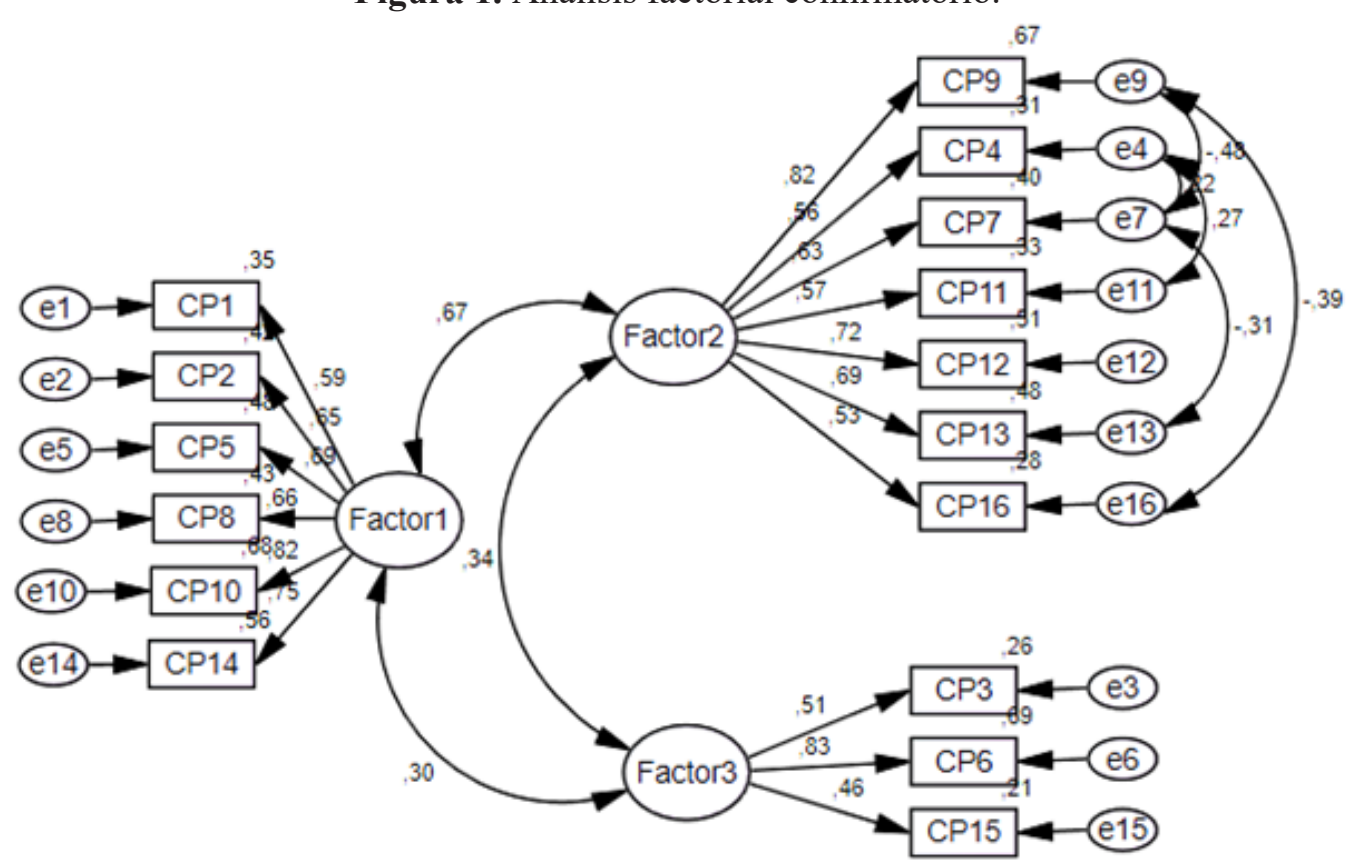

Tabla 3. Estadísticos de ajuste del modelo.

\begin{tabular}{|l|c|c|c|c|c|c|c|c|}
\hline & $\mathbf{X}^{\mathbf{2}}$ & $\mathbf{g l}$ & $\mathbf{X}^{2} / \mathbf{g l}$ & $\mathbf{G F I}$ & AGFI & TLI & CFI & RMSEA \\
\hline Obtenido & 144.533 & \multirow{2}{*}{96} & 1.506 & .882 & .834 & .920 & .936 & .06 \\
\cline { 1 - 6 } Esperado & - & & $<3$ & $>.85$ & $>.85$ & $>.90$ & $>.90$ & $<.1$ \\
\hline
\end{tabular}


Como puede observarse en la Figura 1 y en la Tabla 3 , los índices de regresión del factor a cada reactivo fueron positivos y superiores a 0.4 , siendo en todos los casos mayor que el valor dado por el error y las variables extrañas de cada reactivo. Los indicadores estadísticos oscilaron entre aceptables y adecuados, lo que indica la idoneidad del modelo obtenido. Cabe hacer notar que, en el análisis factorial confirmatorio, el reactivo 9 pasó del factor Valoración general positiva del cuidado al factor Compromiso con el cuidado.

\section{DISCUSIÓN}

El presente estudio tuvo como objetivo diseñar y validar una escala que midiera la actitud hacia los cuidados paliativos de un familiar, puesto que este constructo únicamente ha sido evaluado en profesionales de la salud y no en el núcleo familiar, aun cuando la familia es una parte esencial de la medicina paliativa porque constituye el foco principal de atención para conseguir la armonía y el equilibrio necesarios durante el proceso psicológico que experimenta un enfermo en fase terminal (Tejeda, 2011). Por lo anterior, se consideró relevante crear un instrumento que evaluara las actitudes respecto al cuidado paliativo.

La recopilación teórica muestra que las investigaciones desarrolladas en torno al ámbito de los cuidados paliativos en los profesionales de la salud abarcan cinco pilares, que incluyen la toma de decisiones, la experiencia emocional compartida, la vinculación social, el tiempo de cuidado y la logística (Butow et al., 2007; Ferrer et al., 2016; Padgett y Ferrer, 2015; Rodrigues, 2010). Como complemento de lo anterior, según Briñol et al. (2007), las actitudes se dividen en tres componentes: cognitivo, conductual y afectivo, lo que implica que las actitudes hacia los cuidados paliativos se abarquen en quince componentes que se muestran en la tabla de especificaciones (Apéndice A).

Consecuentemente, en el análisis factorial exploratorio se apreciaron indicadores de extracción apropiados para todos los reactivos, los cuales se agruparon en tres factores que explican 56.49\% de la varianza total acumulada, que si bien no se corresponde con la distribución teóricamente es-

Instituto de Investigaciones Psicológicas - Universidad Veracruzana perada, es coherente respecto a la siguiente conformación: el primer factor, llamado Valoración general positiva del cuidado (reactivos $1,2,5$, $8,10,14)$, el segundo, Compromiso con el cuidado (reactivos $4,7,9,11,12,13,16$ ), y el tercero, denominado Valoración general negativa del cuidado (reactivos 3, 6 y 15). Este modelo de agrupación obtuvo coeficientes de ajuste apropiados para confirmar el modelo, lo que, sumado a los índices de confiabilidad y consistencia interna y a los hallazgos en el modelo confirmatorio, permiten afirmar que la Escala de Actitudes hacia los Cuidados Paliativos de un Familiar es un instrumento válido y confiable para ser utilizado en población colombiana.

Si bien el tamaño muestral de esta investigación fue apropiado a la luz de la potencia obtenida en los estadísticos de muestreo, tuvo algunas limitaciones entre las cuales se incluye que dicha muestra no fue representativa ya que no se obtuvo de forma aleatoria ni puede generalizarse a todo el contexto colombiano, por lo que se recomienda la replicación del presente estudio para garantizar estos hallazgos y, de esta manera, contar con un mayor sustento empírico, lo que permitiría también explicar por qué algunos de los estadísticos del análisis confirmatorio fueron aceptables.

Respecto a la utilidad clínica de la escala, se encuentran diversos beneficios en su implementación, pues proporciona una medida de tamizaje que hace posible identificar al mejor cuidador para el paciente en términos de su compromiso con el cuidado y la valoración que hace del mismo, lo cual se encuentra justificado por los índices de validez y confiabilidad de la prueba. Lo anterior posibilita la creación de programas orientados a desarrollar habilidades para el cuidado paliativo en el caso de que se obtenga una puntuación que denote una actitud negativa o muy negativa hacia aquel.

Finalmente, teniendo en cuenta que en la revisión teórica no se encontraron instrumentos que midieran el constructo "Actitudes hacia los cuidados paliativos de un familiar" en población colombiana, es posible afirmar que el presente trabajo puede considerarse pionero en este aspecto, por lo cual se recomienda extender la investigación a cuidadores potenciales y a familiares de pacientes 
que se encuentren ejerciendo dicho rol, así como evaluar la existencia de variables asociadas y predictoras, e indagar la posible existencia de corre- laciones entre la actitud del cuidador (cuando es un familiar) y la evolución del paciente.

Citación: Pedroza V., T., Garzón R., N., Ortíz B., P., Murcia L., D. y Riveros M., F. (2021). Diseño y validación de la Escala de Actitudes hacia los Cuidados Paliativos de un Familiar. Psicología y Salud, 32(1), 25-38. https://doi.org/10.25009/pys.v32i1.2708.

\section{REFERENCIAS}

American Psychological Association (1992). Ethical principles of psychologists and code of conduct. American Psychologist, 47, 1597-1611.

Arriaza, P., Cancino, G. y Sanhueza, O. (2009). Pertenecer a algo mayor: experiencias de pacientes y cuidadores durante el cuidado paliativo en Chile. Ciencia y Enfermería, 15(2), 95-106.

Ascencio, L. (2015). Adaptación en español de la Escala de Actitudes ante Cuidados Paliativos: confiabilidad y análisis factorial. Psicooncología, 12(2/3), 367-381. Doi: 10.5209/rev_PSIC.2015.v12.n2-3.51015.

Ascencio, L., Allende, S.R. y Verástegui, E. (2014). Creencias, actitudes y ansiedad ante la muerte en un equipo multidisciplinario de cuidados paliativos oncológicos. Psicooncología, 11(1), 101-115. Doi: 10.5209/rev_PSIC.2014.v11.n1.44920.

Bermejo, J.C., Villacieros, M., Carabias, R., Sánchez, E. y Díaz, B. (2013). Conspiración del silencio en familiares y pacientes al final de la vida ingresados en una unidad de cuidados paliativos: nivel de información y actitudes observadas. Medicina Paliativa, 20(2), 49-59.

Briñol, P., Falces, C. y Becerra, A. (2007). Actitudes. En J. Morales, E. Gaviria, M. Moya y M. Cuadrado (Ed.): Psicología social (pp. 457-490). Madrid: McGraw-Hill.

Butow, P., Carrick, S., Clayton, J., Currow, D., Ghersi, D., Glare, P., Hagerty, R., Hancock, K., Parker, S., Tattersall, M. y Walder, S. (2007). A systematic review of prognostic/end-of-life communication with adults in the advanced stages of a life-limiting illness: Patient/caregiver preferences for the content, style, and timing of information. Journal of Pain and Symptom Management, 34(1), 81-93.

Escobar T., J. (1998). El morir como ejercicio final del derecho a una vida digna. Buenos Aires: Ediciones El Bosque.

Espinoza, M., Luengo, L. y Sanhueza, O. (2016). Actitudes en profesionales de enfermería chilenos hacia el cuidado al final de la vida. Análisis multivariado. Aquichán, 16(4), 430-446.

Ferrando, P.J. y Anguiano C., C. (2010). El análisis factorial como técnica de investigación en psicología. Papeles del Psicólogo, 31(1), 18-33.

Ferrer, R., Padgett, L. y Ellis, E. (2016). Extending emotion and decision-making beyond the laboratory: The promise of palliative care contexts. Emotion, 16(5), 581-586.

Flores E., G., Rivas, E. y Seguel, F. (2012). Nivel de sobrecarga en el desempeño del rol del cuidador familiar de adulto mayor con dependencia severa. Ciencia y Enfermería, 18(1), 29-41.

Gómez, C., Clemente, M., Bugallo, C. y Gandoy, M. (2016). Cuidados paliativos gerontológicos: influencia de las condiciones laborales y burnout en el personal de enfermería. Gerokomos, 27(3), 91-96.

Grau, J., Llantá M., C., Massip, C., Chacón, M., Reyes, M.C., Infante O., S., Romero, T., Barroso, I. y Morales, D. (2008). Ansiedad y actitudes ante la muerte: revisión y caracterización en un grupo heterogéneo de profesionales que se capacita en cuidados paliativos. Pensamiento Psicológico, 4(10), 27-58.

Lloret, S., Ferreres, A., Hernández, A. y Tomás, I. (2014). El análisis factorial exploratorio de los ítems: una guía práctica, revisada y actualizada. Anales de Psicología, 30(3), 1151-1169.

López, J. (2015). Encuesta de actitudes y conocimientos médicos en el manejo paliativo hospitalario. Revista de Ciencias de la Salud, 14(1), 53-61.

Marchán, S. (2016). Afrontamiento del profesional de enfermería ante la muerte de pacientes en unidades de paliativos y oncología. Nure Investigación, 13(82), 1-12.

Maslach, C. y Jackson, S.E. (1981). The measurement of experienced burnout. Journal of Organizational Behavior, 2(2), 99-113.

Ministerio de Salud de Colombia (1993). Resolución Número 8430: Normas científicas, técnicas y administrativas para la investigación en salud. Bogotá: Autor.

Montero, I. y León, O. (2005). Sistema de clasificación del método en los informes de investigación en Psicología. International Journal of Clinical and Health Psychology, 5(1). Recuperado de http://www.redalyc.org/pdf/337/33701007.pdf. 
Observatorio Colombiano de Cuidados Paliativos (2016). ¿Cómo vamos en cuidados paliativos en Colombia? Bogotá: Autor.

Padgett, L. y Ferrer, R. (2015). Palliative care in cancer: Enhancing our view with the science of emotion and decision making. Journal of Palliative Medicine, 18(6), 479-479.

Pastrana, T., de Lima, L. y Wenk, R. (2015). Estudio multicéntrico sobre la comodidad y el interés en cuidados paliativos en estudiantes de pregrado en Colombia. Medicina Paliativa, 22(4), 136-145.

Rodrigues, A. (2010). El cuidador y el enfermo en el final de la vida, familia y/o persona significativa. Enfermería Global, 18. Recuperado de http://scielo.isciii.es/scielo.php?script=sci_arttextypid=S1695-61412010000100022ylng=esytlng=es.

Sánchez, J., Morales, J., Morales, I., Sánchez, J., Timonet, E. y Rivas, F. (2017). Análisis de la toma de decisiones compartidas al final de la vida en las historias clínicas. Medicina Paliativa, 24(1), 14-20.

Sarmiento, M. (2015). El cuidado paliativo: un recurso para la atención del paciente con enfermedad terminal. Revista Salud Bosque, 1(2), 23-37.

Tejeda, Y. (2011). La familia como agente terapéutico en los cuidados paliativos. Medisan, 15(2), 197-203.

Tripodoro, V., Veloso, V. y Llanos, V. (2015). Sobrecarga del cuidador principal de pacientes en cuidados paliativos. Argumentos. Revista de Crítica Social, 17. Recuperado de http://publicaciones.sociales.uba.ar/index.php/argumentos/article/view/1324. 
Apéndice A. Tabla de especificaciones.

\begin{tabular}{|c|c|c|c|c|}
\hline & SUBESCALAS & $\begin{array}{l}\text { Componente cognitivo } \\
37.5 \% \text {, quince reactivos. } \\
\text { Incluye los pensamientos } \\
\text { y creencias que la perso- } \\
\text { na tiene acerca del objeto } \\
\text { de actitud. }\end{array}$ & $\begin{array}{l}\text { Componente afectivo } \\
25 \% \text {, diez reactivos. } \\
\text { Sentimientos y emociones } \\
\text { asociados al objeto de } \\
\text { actitud. }\end{array}$ & $\begin{array}{l}\text { Componente conductual } \\
37.5 \%, 15 \text { reactivos. } \\
\text { Intenciones, disposiciones } \\
\text { a la acción y comporta- } \\
\text { mientos dirigidos hacia el } \\
\text { objeto de actitud. }\end{array}$ \\
\hline \multirow{5}{*}{$\begin{array}{l}0 \\
0 \\
\vdots \\
\vdots \\
0 \\
0 \\
0 \\
0 \\
0 \\
0 \\
0 \\
0 \\
0\end{array}$} & $\begin{array}{l}\text { Toma de decisiones } \\
20 \% \text {, ocho reactivos. } \\
\text { Las decisiones relaciona- } \\
\text { das al proceso asistencial } \\
\text { en curso, tomadas por } \\
\text { familiares. }\end{array}$ & $\begin{array}{l}7.5 \% \text {, tres reactivos. } \\
\text { Pensamientos y creencias } \\
\text { respecto a la respon- } \\
\text { sabilidad de tener a un } \\
\text { familiar en cuidados } \\
\text { paliativos. }\end{array}$ & $\begin{array}{l}5 \%, \text { dos reactivos. } \\
\text { Sentimientos y emocio- } \\
\text { nes involucrados con la } \\
\text { responsabilidad de tener } \\
\text { a un familiar en cuidados } \\
\text { paliativos. }\end{array}$ & $\begin{array}{l}7.5 \% \text {, tres reactivos. } \\
\text { Disposiciones e intencio- } \\
\text { nes a responsabilizarse } \\
\text { en la toma de decisiones } \\
\text { con respecto al proceso } \\
\text { asistencial. }\end{array}$ \\
\hline & $\begin{array}{l}\text { Experiencia emocional } \\
\text { compartida } \\
20 \%, 8 \text { reactivos. } \\
\text { Experiencia emocional } \\
\text { en la persona que toma la } \\
\text { decisión. }\end{array}$ & $\begin{array}{l}7.5 \%, 3 \text { reactivos. } \\
\text { Pensamientos y creencias } \\
\text { respecto a las emociones } \\
\text { que intervienen en el } \\
\text { cuidado paliativo de un } \\
\text { familiar. }\end{array}$ & $\begin{array}{l}5 \%, 2 \text { reactivos. } \\
\text { Sentimientos y emociones } \\
\text { asociados al cuidado palia- } \\
\text { tivo de un familiar. }\end{array}$ & $\begin{array}{l}7.5 \%, 3 \text { reactivos. } \\
\text { Disposiciones a los com- } \\
\text { portamientos emocionales } \\
\text { y afectivos hacia un fami- } \\
\text { liar en cuidados paliativos. }\end{array}$ \\
\hline & $\begin{array}{l}\text { Vinculación social } \\
20 \% \text {, ocho reactivos. } \\
\text { Las emociones subyacen- } \\
\text { tes de las relaciones inter- } \\
\text { personales preexistentes } \\
\text { y se conciben como las } \\
\text { relaciones que componen } \\
\text { el entorno social del pa- } \\
\text { ciente, las cuales atravie- } \\
\text { san cualquier proceso en } \\
\text { el que este se involucre. }\end{array}$ & $\begin{array}{l}7.5 \% \text {, tres reactivos. } \\
\text { Pensamientos y creencias } \\
\text { sobre las relaciones inter- } \\
\text { personales preexistentes } \\
\text { de un familiar en relación } \\
\text { con su proceso de cuida- } \\
\text { dos paliativos. }\end{array}$ & $\begin{array}{l}5 \% \text {, dos reactivos. } \\
\text { Sentimientos y emociones } \\
\text { respecto a las relaciones } \\
\text { interpersonales preexisten- } \\
\text { tes de un familiar a causa } \\
\text { de su proceso de cuidados } \\
\text { paliativos. }\end{array}$ & $\begin{array}{l}7.5 \%, \text { tres reactivos. } \\
\text { Disposiciones e inten- } \\
\text { ciones involucradas con } \\
\text { el mantenimiento de } \\
\text { relaciones preexistentes } \\
\text { de un familiar en cuidados } \\
\text { paliativos. }\end{array}$ \\
\hline & $\begin{array}{l}\text { Tiempo del cuidado } \\
\text { 20\%, ocho reactivos. } \\
\text { Tiempo invertido para el } \\
\text { cuidado diario y la pro- } \\
\text { longación cronológica que } \\
\text { dicho cuidado tendrá. }\end{array}$ & $\begin{array}{l}\text { 7.5\%, tres reactivos. } \\
\text { Pensamientos y creen- } \\
\text { cias sobre el tiempo que } \\
\text { deben emplear en el } \\
\text { cuidado paliativo de un } \\
\text { familiar enfermo. }\end{array}$ & $\begin{array}{l}5 \%, \text { dos reactivos. } \\
\text { Sentimientos y emociones } \\
\text { generados por el tiempo } \\
\text { que se debe emplear en el } \\
\text { cuidado paliativo de un } \\
\text { familiar enfermo. }\end{array}$ & $\begin{array}{l}7.5 \% \text {, tres reactivos. } \\
\text { Disposiciones e intencio- } \\
\text { nes relacionados con el } \\
\text { tiempo a emplear en el } \\
\text { cuidado y acompañamien- } \\
\text { to de un familiar enfermo. }\end{array}$ \\
\hline & $\begin{array}{l}\text { Logística } \\
20 \% \text {, ocho reactivos. } \\
\text { Planeación, Distribución } \\
\text { y ejecución de las tareas y } \\
\text { actividades necesarias por } \\
\text { parte del cuidador en los } \\
\text { cuidados paliativos. }\end{array}$ & $\begin{array}{l}7.5 \% \text {, tres reactivos. } \\
\text { Pensamientos y creencias } \\
\text { acerca de la planeación } \\
\text { de tareas necesarias en el } \\
\text { cuidado paliativo. }\end{array}$ & $\begin{array}{l}5 \%, \text { dos reactivos. } \\
\text { Sentimientos y emociones } \\
\text { involucradas en la planea- } \\
\text { ción de actividades del } \\
\text { cuidado paliativo. }\end{array}$ & $\begin{array}{l}7.5 \%, \text { tres reactivos. } \\
\text { Disposiciones e inten- } \\
\text { ciones asociadas a la } \\
\text { ejecución de actividades } \\
\text { necesarias en el cuidado } \\
\text { paliativo. }\end{array}$ \\
\hline
\end{tabular}


Apéndice B. Índice de validez de contenido de Lawshe.

\begin{tabular}{|c|c|c|c|c|c|}
\hline Ítem & Pertinencia & Coherencia & Redacción & Objetividad & Decisión \\
\hline 1 & $<0.6$ & $>0.6$ & $<0.6$ & $<0.6$ & SD \\
\hline 2 & $>0.6$ & $>0.6$ & $>0.6$ & $>0.6$ & SM \\
\hline 3 & $>0.6$ & $>0.6$ & $>0.6$ & $>0.6$ & SM \\
\hline 4 & $>0.6$ & $>0.6$ & $>0.6$ & $>0.6$ & SM \\
\hline 5 & $<0.6$ & $<0.6$ & $<0.6$ & $<0.6$ & SD \\
\hline 6 & $>0.6$ & $>0.6$ & $>0.6$ & $>0.6$ & SM \\
\hline 7 & $<0.6$ & $<0.6$ & $<0.6$ & $<0.6$ & SD \\
\hline 8 & $<0.6$ & $<0.6$ & $<0.6$ & $<0.6$ & SD \\
\hline 9 & $>0.6$ & $>0.6$ & $>0.6$ & $>0.6$ & SM \\
\hline 10 & $>0.6$ & $>0.6$ & $>0.6$ & $>0.6$ & $\mathrm{SC}$ \\
\hline 11 & $>0.6$ & $>0.6$ & $>0.6$ & $>0.6$ & SM \\
\hline 12 & $>0.6$ & $>0.6$ & $>0.6$ & $>0.6$ & SM \\
\hline 13 & $>0.6$ & $>0.6$ & $>0.6$ & $>0.6$ & SM \\
\hline 14 & $>0.6$ & $>0.6$ & $>0.6$ & $>0.6$ & SM \\
\hline 15 & $>0.6$ & $>0.6$ & $>0.6$ & $>0.6$ & SM \\
\hline 16 & $>0.6$ & $>0.6$ & $>0.6$ & $>0.6$ & SM \\
\hline 17 & $>0.6$ & $>0.6$ & $>0.6$ & $>0.6$ & SM \\
\hline 18 & $>0.6$ & $>0.6$ & $>0.6$ & $>0.6$ & SM \\
\hline 19 & $>0.6$ & $>0.6$ & $>0.6$ & $>0.6$ & SM \\
\hline 20 & $>0.6$ & $>0.6$ & $>0.6$ & $>0.6$ & SM \\
\hline 21 & $>0.6$ & $>0.6$ & $>0.6$ & $>0.6$ & SM \\
\hline 22 & $>0.6$ & $>0.6$ & $>0.6$ & $>0.6$ & SM \\
\hline 23 & $>0.6$ & $>0.6$ & $>0.6$ & $>0.6$ & SM \\
\hline 24 & $>0.6$ & $>0.6$ & $>0.6$ & $>0.6$ & SM \\
\hline 25 & $>0.6$ & $>0.6$ & $>0.6$ & $>0.6$ & SM \\
\hline 26 & $>0.6$ & $>0.6$ & $>0.6$ & $>0.6$ & SM \\
\hline 27 & $>0.6$ & $>0.6$ & $>0.6$ & $>0.6$ & SM \\
\hline 28 & $>0.6$ & $>0.6$ & $>0.6$ & $>0.6$ & $\mathrm{SC}$ \\
\hline 29 & $>0.6$ & $>0.6$ & $>0.6$ & $>0.6$ & SM \\
\hline 30 & $>0.6$ & $>0.6$ & $>0.6$ & $>0.6$ & SM \\
\hline 31 & $>0.6$ & $>0.6$ & $>0.6$ & $>0.6$ & SM \\
\hline 32 & $>0.6$ & $>0.6$ & $>0.6$ & $>0.6$ & $\mathrm{SM}$ \\
\hline 33 & $>0.6$ & $>0.6$ & $>0.6$ & $>0.6$ & SM \\
\hline 34 & $>0.6$ & $>0.6$ & $>0.6$ & $>0.6$ & SM \\
\hline 35 & $>0.6$ & $>0.6$ & $>0.6$ & $>0.6$ & SM \\
\hline 36 & $>0.6$ & $>0.6$ & $>0.6$ & $>0.6$ & SM \\
\hline 37 & $>0.6$ & $>0.6$ & $>0.6$ & $>0.6$ & SM \\
\hline 38 & $>0.6$ & $>0.6$ & $>0.6$ & $>0.6$ & $\mathrm{SC}$ \\
\hline 39 & $>0.6$ & $>0.6$ & $>0.6$ & $>0.6$ & SM \\
\hline 40 & $>0.6$ & $>0.6$ & $>0.6$ & $>0.6$ & $\mathrm{SC}$ \\
\hline 41 & $>0.6$ & $>0.6$ & $>0.6$ & $>0.6$ & SM \\
\hline 42 & $>0.6$ & $>0.6$ & $>0.6$ & $>0.6$ & SM \\
\hline
\end{tabular}

Continúa... 


\begin{tabular}{|l|l|l|l|l|l|}
\hline 43 & $>0.6$ & $>0.6$ & $>0.6$ & $>0.6$ & $\mathrm{SM}$ \\
\hline 44 & $>0.6$ & $>0.6$ & $>0.6$ & $>0.6$ & $\mathrm{SM}$ \\
\hline 45 & $>0.6$ & $>0.6$ & $>0.6$ & $>0.6$ & $\mathrm{SC}$ \\
\hline 46 & $>0.6$ & $>0.6$ & $>0.6$ & $>0.6$ & $\mathrm{SM}$ \\
\hline 47 & $>0.6$ & $>0.6$ & $>0.6$ & $>0.6$ & $\mathrm{SM}$ \\
\hline 48 & $<0.6$ & $<0.6$ & $>0.6$ & $>0.6$ & $\mathrm{SD}$ \\
\hline 49 & $>0.6$ & $>0.6$ & $>0.6$ & $>0.6$ & $\mathrm{SM}$ \\
\hline 50 & $<0.6$ & $<0.6$ & $<0.6$ & $<0.6$ & $\mathrm{SD}$ \\
\hline 51 & $>0.6$ & $>0.6$ & $>0.6$ & $>0.6$ & $\mathrm{SC}$ \\
\hline 52 & $>0.6$ & $>0.6$ & $>0.6$ & $>0.6$ & $\mathrm{SM}$ \\
\hline 53 & $>0.6$ & $>0.6$ & $>0.6$ & $>0.6$ & $\mathrm{SM}$ \\
\hline 54 & $>0.6$ & $>0.6$ & $>0.6$ & $>0.6$ & $\mathrm{SM}$ \\
\hline 55 & $>0.6$ & $>0.6$ & $>0.6$ & $>0.6$ & $\mathrm{SM}$ \\
\hline 56 & $>0.6$ & $>0.6$ & $>0.6$ & $>0.6$ & $\mathrm{SM}$ \\
\hline 57 & $>0.6$ & $>0.6$ & $>0.6$ & $>0.6$ & $\mathrm{SM}$ \\
\hline 58 & $>0.6$ & $>0.6$ & $>0.6$ & $>0.6$ & $\mathrm{SM}$ \\
\hline 59 & $>0.6$ & $>0.6$ & $>0.6$ & $>0.6$ & $\mathrm{SM}$ \\
\hline 60 & $>0.6$ & $>0.6$ & $>0.6$ & $>0.6$ & $\mathrm{SM}$ \\
\hline 61 & $>0.6$ & $>0.6$ & $>0.6$ & $>0.6$ & $\mathrm{SM}$ \\
\hline 62 & $>0.6$ & $>0.6$ & $>0.6$ & $>0.6$ & $\mathrm{SM}$ \\
\hline $\mathrm{SD}$ & $>0$ & $>6$ & & \\
\hline
\end{tabular}

$\mathrm{SD}=$ Se desecha; $\mathrm{SM}=$ Se modifica; $\mathrm{SC}=$ Se conserva .

Apéndice C. Índice de concordancia W-Kendall.

\begin{tabular}{|l|c|c|}
\cline { 2 - 3 } \multicolumn{1}{c|}{} & W- Kendall & $\boldsymbol{p}$ \\
\hline Pertinencia & 0.300 & 0.007 \\
\hline Coherencia & 0.303 & 0.006 \\
\hline Redacción & 0.351 & $<0.001$ \\
\hline Objetividad & 0.367 & $<0.001$ \\
\hline
\end{tabular}

Apéndice D. Cuestionario para las actitudes hacia los cuidados paliativos de familiares.

Nombre:

Edad: Sexo:

Lea atentamente la siguiente información y con base en esta responda a cada afirmación.

En la escala, los cuidados paliativos se entienden como aquellas actividades involucradas en el acompañamiento de un familiar que padece una enfermedad progresiva, avanzada e incurable. Ahora, imagine que un familiar que usted considere cercano requiere este tipo de cuidados.

Tenga presente que no existe una respuesta más acertada que otra. Es probable que en algunos casos le sea más difícil elegir la respuesta; sin embargo, seleccione la opción que más se acomode a usted, y no deje espacios en blanco.

A continuación se le presentarán una serie de afirmaciones para las cuales usted deberá valorar de 1 a 4 según la siguiente escala: 1) Totalmente desacuerdo; 2) En desacuerdo; 3) De acuerdo; 4) Totalmente de acuerdo. 


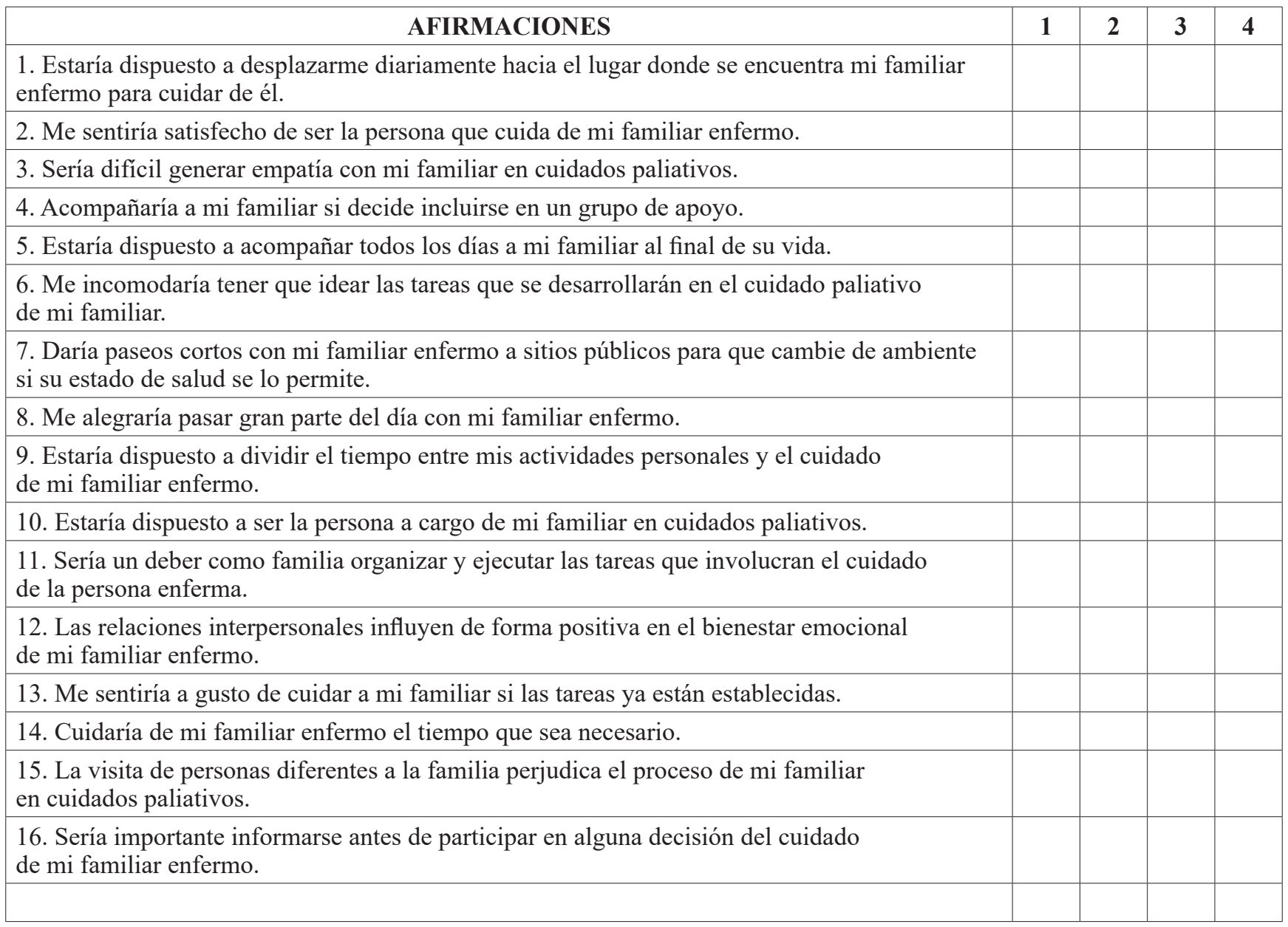

Apéndice E. Normas de calificación.

Para la evaluación de la escala de actitudes hacia los cuidados paliativos de un familiar, se tienen en cuenta tres dimensiones: (a) Evaluación general positiva hacia el cuidado, que incluye los reactivos 1, 2, 5, 8, 10, 14; (b) Compromiso con el cuidado, que incluye los reactivos 4, 7, 9, 11, 12, 13 y 16, y (c) Evaluación generalmente negativa hacia el cuidado, que incluye los ítems 3, 6, 15 .

Además, se tendrá en cuenta el siguiente método de calificación para cada ítem:

(a) Los ítems: 1, 2, 4, 5, 7, 8, 9, 10, 11, 12, 13, 14 y 16 se calificarán teniendo en cuenta que la respuesta "Totalmente de acuerdo" equivale a 4 puntos, y la respuesta "Totalmente en desacuerdo" equivale a 1 punto.

(b) Los ítems 3, 6 y 15 se calificarán teniendo en cuenta que la respuesta "Totalmente de acuerdo" equivale a 1 punto, y la respuesta "Totalmente en desacuerdo" equivale a 4 puntos.

Los puntajes se deben sumar teniendo en cuenta que, como mínimo puntaje, es posible obtener 16 puntos y, como máximo, 64. De acuerdo con el total de dicha suma, los individuos se clasificarán tal y como se muestra en la siguiente tabla. 


\begin{tabular}{|l|c|c|c|c|}
\hline \multicolumn{1}{|c|}{ Actitudes } & $\begin{array}{c}\text { Valoración general } \\
\text { positiva hacia el cuidado }\end{array}$ & $\begin{array}{c}\text { Compromiso } \\
\text { con el cuidado }\end{array}$ & $\begin{array}{c}\text { Valoración general negativa } \\
\text { hacia el cuidado }\end{array}$ & Puntaje total \\
\hline $\begin{array}{l}\text { Actitud } \\
\text { muy negativa }\end{array}$ & $7-11$ & $6-10$ & $3-5$ & $16-26$ \\
\hline Actitud negativa & $12-17$ & $11-15$ & $6-7$ & $26-39$ \\
\hline Actitud positiva & $18-23$ & $16-20$ & $8-9$ & $40-52$ \\
\hline $\begin{array}{l}\text { Actitud } \\
\text { muy positiva }\end{array}$ & $24-28$ & $21-24$ & $10-12$ & $53-64$ \\
\hline
\end{tabular}

